\title{
CORRECTION
}

\section{Correction: Study of structures and thermodynamics of CuNi nanoalloys using a new DFT-fitted atomistic potential}

Cite this: Phys. Chem Chem. Phys. $2015,17,28407$

DOI: $10.1039 / c 5 c p 90107 c$

www.rsc.org/pccp

\author{
Emanuele Panizon, ${ }^{a}$ Jimena A. Olmos-Asar, ${ }^{b}$ Maria Peressi ${ }^{b}$ and \\ Riccardo Ferrando*c
}

Correction for 'Study of structures and thermodynamics of CuNi nanoalloys using a new DFT-fitted atomistic potential' by Emanuele Panizon et al., Phys. Chem. Chem. Phys., 2015, DOI: 10.1039/c5cp00215j.

In the published version of the article, there are a couple of errors in Table 1 . The corrected version can be found below:

Table 1 Bulk values for $\mathrm{Cu}$ and Ni obtained with DFT simulations and parameter sets of the potential. $a$ is the lattice parameter, $E_{\mathrm{c}}$ is the cohesive energy per atom, $B$ is the bulk modulus and $\Delta E_{\mathrm{hcp} \text {-fcc }}$ is the difference in binding energy per atom between hcp and fcc bulk phases

\begin{tabular}{lllll}
\hline & $a(\AA)$ & $E_{\mathrm{c}}(\mathrm{eV})$ & $B(\mathrm{GPa})$ & $\Delta E_{\mathrm{hcp}-\mathrm{fcc}}(\mathrm{eV})$ \\
\hline $\mathrm{Cu}$ & 3.649 & -3.429 & 138.7 & 0.011 \\
$\mathrm{Ni}$ & 3.518 & -4.931 & 206.6 & 0.031 \\
\hline & $p$ & $q$ & $(\mathrm{eV})$ & $\xi(\mathrm{eV})$ \\
\hline $\mathrm{Cu}-\mathrm{Cu}$ & 10.653 & 2.49 & 0.092585 & 1.2437 \\
$\mathrm{Ni}-\mathrm{Ni}$ & 11.7 & 2.045 & 0.1046 & 1.6111 \\
$\mathrm{Cu}-\mathrm{Ni}$ & 11.1765 & 2.2675 & $0.194 \mathrm{eV}$ \\
\hline$E_{\mathrm{s}}$ Cu-impurity in Ni bulk & & & $0.113 \mathrm{eV}$ \\
$E_{\mathrm{s}}$ Ni-impurity in Cu bulk & & & & 1.4453 \\
\hline
\end{tabular}

The Royal Society of Chemistry apologises for these errors and any consequent inconvenience to authors and readers.

\footnotetext{
${ }^{a}$ Physics Department, University of Genoa, Via Dodecaneso 33, 16146, Genoa, Italy

${ }^{b}$ Department of Physics, University of Trieste, Strada Costiera 11, 34151 Trieste, Italy

${ }^{c}$ Physics Department, University of Genoa and CNR-IMEM, Via Dodecaneso 33, 16146, Genoa, Italy. E-mail: ferrando@fisica.unige.it
} 